\title{
Effect of training on the ability of dual-task coordination
}

F.M. Rosin,

R.P. Sylwan

and C. Galera

\section{Correspondence \\ R.P. Sylwan \\ Laboratório de Psicologia \\ Experimental Humana \\ Departamento de Psicologia e \\ Educação, FFCLRP, USP \\ 14040-901 Ribeirão Preto, SP \\ Brasil \\ Fax: + 55-16-633-6361 \\ E-mail: rpsylwan@usp.br}

Part of these data were presented at the XIII Annual M eeting of the

Federação de Sociedades de Biologia Experimental, Caxambu, MG, Brasil, August 26-29, 1998, at the XVII Congresso Brasileiro de Neurologia, São Paulo, SP, Brasil, August 29September 3, 1998 and at the II Congreso Latinoamericano/l Congreso Argentino de Neuropsicofarmacología, Buenos Aires, Argentina, September 16-19, 1998.

F.M. Rosin and R.P. Sylwan are recipients of CAPES/SPU fellowships. Publication supported by FAPESP. The first two authors played equal roles in the research and publication of this study.

Received O ctober 15, 1998 Accepted August 19, 1999

\author{
Laboratório de Psicologia Experimental Humana, \\ Departamento de Psicologia e Educação, Faculdade de Filosofia, \\ Ciências e Letras de Ribeirão Preto, U niversidade de São Paulo, \\ Ribeirão Preto, SP, Brasil
}

\section{Abstract}

Within the framework of the working memory model proposed by A. Baddeley and G. Hitch, a dual-task paradigm has been suggested to evaluate the capacity to perform simultaneously two concurrent tasks. This capacity is assumed to reflect the functioning of the central executive component, which appears to be impaired in patients with dysexecutive syndrome. The present study extends the investigation of an index (" $m u$ "), which is supposed to indicate the capacity of coordination of concurrent auditory digit span and tracking tasks, by testing the influence of training on the performance in the dual task. The presentation of the same digit sequence lists or always-different lists did not differently affect the performance. The span length affected the $m u$ values. The improved performance in the tasks under the dual condition closely resembled the improvement in the singletask performance. So, although training improved performance in the single and dual conditions, especially for the tracking component, the $m u$ values remained stable throughout the sessions when the single tasks were performed first. Conversely, training improved the capacity of dual-task coordination throughout the sessions when dual task was performed first, addressing the issue of the contribution of the withinsession practice to the $m u$ index.

\section{Introduction}

The working memory (WM) model, formulated by Baddeley and Hitch (1), aims to explain the processes and structures of shortterm memory (STM) and to study the holding and manipulation of information during the performance of several cognitive tasks, such as comprehension, learning and reasoning. The need to explain the capacity to hold a concurrent load of items in reasoning tasks, without impairing anyone of them, led Baddeley and Hitch to propose a verbal subsystem called phonological loop (PL). According to the WM model, the PL is differen- tiated from the visuospatial sketchpad (VS), which allows to keep and to manipulate the visuospatial information. VS and PL are coordinated by a central executive (CE), which despite its importance has been the subject of a relatively small number of studies (2-5).

Two components may be differentiated in the VS subsystem: the "passive storage" and the "active imagery" (6). The task of generating images suffers interference from another visuospatial task, such as pointing to a moving target. This last task utilizes part of the capacity of the image generation mechanisms, leaving less free capacity available for other visual tasks; but this kind of task 
does not interfere with verbal tasks (4).

The phonological similarity, articulatory suppression, word length and irrelevant speech effects on the STM constitute the more relevant experimental phenomena for the proposal of the PL. This subsystem consists of two principal components: a subvocal rehearsal and a phonological short-term store. The subvocal rehearsal allows to refresh the transient phonological storage contents and to convert the visually presented verbal stimuli to a phonological code (5,7-9).

The verbal short-term memory span is supposed to determine the storage capacity of the PL. Performance in span tasks is supposed to involve limitations of capacity and duration. The number of items that can be retained and reproduced exactly following a single presentation expresses limitation of capacity. The rate at which material is forgotten without possibility of rehearsal expresses limitation of duration (10). The immediate memory span was traditionally interpreted as evidence of the storage capacity defined by a small number of items or "chunks". However, the effect of word length may be considered as evidence against the view that memory span consists of a constant number of items. Memory span is related to the efficiency of the PL-based rehearsal processes by refreshing the phonological storage contents and allowing more items to be kept active at the same time when stimuli are encoded phonologically $(5,9)$. Memoryspan differences are also explained by Cowan et al. (11) by means of the contribution of the $\mathrm{CE}$ mechanisms to the rehearsal strategies through the rate of retrieval process.

Functions like complex strategy selection, planning and control of the recovery of stored information have been attributed to the CE. The executive system directs response sequences and the flow of thoughts, making possible the achievement of behavioral goals $(3,12-14)$. The coordination of separate tasks, the capacity of switching strategies of information recall, the capacity of selective attention and the temporary hold- ing and manipulation of information from long-term memory have been considered as CE functions (2).

The requirement to perform two tasks simultaneously involves resources other than those required for the same tasks executed separately. The subjects' performance under the dual-task condition might be impaired because of heavy loads on their capacities in the single task (15-17). Studies of performance in simultaneous verbal and tracking tasks have shown that tracking error was a direct function of the difficulty of the verbal task, especially when recall rather than encoding was involved (18). The capacity to perform two tasks simultaneously seems to be an isolated characteristic of the CE. Age does not seem to affect significantly this capacity, whereas it appears to affect other executive functions; this could imply the fractionating of the CE into different subcomponents (2). The performance in the dual task should not be taken as an indicator of the WM capacity or of the subjects' physical abilities per se, but should be considered as the aptitude to distribute the memory capacity resources (19).

The capacity of coordination of visuospatial and verbal information has been studied using a dual task that combines a tracking task and an auditory digit-span task. It was found that the performance in the simultaneous execution of the tasks was impaired in patients who presented the dysexecutive syndrome, suggesting that impairment in the simultaneous performance in the verbal and visual tasks reflects a common contribution of the CE to the VS and the PL (2,20-23). In a normative study, Baddeley et al. (19) found that the performance in a paper-and-pencil version of the dual task was not affected by age, gender or educational level although the clinical use of the dual task was still limited by its low test-retest reliability. The issue of the attention distribution between tasks for the best possible performance in both tasks was also addressed. Besides the problem 
about the low test-retest reliability of the dual task, we consider that to know the effect of its repeated application on the same subjects is important for the clinical use of the dual task, i.e., for follow-up of the patients' clinical evolution.

In the present study, we addressed the effect of training on the ability of dual-task coordination using the dual task proposed by Della Sala et al. (22). In perceptual-motor tasks, training is accompanied by an increase in accuracy, speed and smoothness of performance, eliminating the need to use the feedback necessary for successful execution and reducing the interference in dual-task performance via automation $(24,25)$. However, training does not prevent from interference with dual tasks, even when training in single tasks reaches some criterion of automaticity (e.g. fastness, effortlessness, autonomy, stereotypy, unconsciousness), and is not limited by STM capacity $(26,27)$. In the present study subjects were asked to perform the dual task throughout 20 sessions. Schwartz and Bryden (28) reported that recall of digit sequences improved with training when the sequences were the same, but not when they were non-repeating sequences, or when the first digits of the repeating sequences were changed. We also attempted to determine whether the composition of the digit sequences would affect the training in the digit task. Thus, subjects were divided into two groups, one of them receiving different digit sequence lists in each session and the other always receiving the same two lists (the dual-task list differed from the single-task list). Although several studies have reported that training in the isolated single tasks does not necessarily improve the dual-task performance $(27,29)$, we also attempted to evaluate the possible improvement in dual-task performance acquired in the execution of the isolated single task. Demands on the control and coordinating functions of $\mathrm{CE}$ appear to be greater when the tasks are novel than after practice in the single tasks (30).

In the direct order of the tasks, as proposed by Della Sala et al. (22), the single tasks precede the dual task, so the practice in the single tasks could be acting on the dual task, masking the real capacity of coordination of novel tasks. Thus, a third group of subjects was asked to perform the dual task before the single tasks throughout 5 sessions.

\section{Material and Methods}

We used a paper-and-pencil version of the tracking test, the box-crossing, combined with the digit-span task to evaluate the executive functions. The digit-span task consists of the repetition of sequences of digits and the box-crossing task consists of traversing a chain of squares with crosses. The dual task consists of the simultaneous execution of both tasks and the performance is estimated by " $m u$ ", a composite index that is supposed to reflect the subject's capacity to coordinate the separate tasks $(19,22,31,32)$.

To estimate $m u$, the performance is evaluated in each task for both the single and dual conditions. The number of digit sequences presented is named $N s$ and $N d$ for the singleand dual-task conditions, respectively. The number of correct digit sequences repeated for the same conditions is defined as $n s$ and $n d$. The performance is indicated by $p s$ and $p d$, and the percentage of correct responses in the single and dual conditions is calculated by the following formulas (19): $p s=n s$ / $N s$ and $p d=n d / N d$. In the box-crossing task, the number of squares correctly traversed with " $\mathrm{X}$ " is named $t s$ and $t d$ for the singleand dual-task conditions, respectively. The proportional memory loss $(\mathrm{pm})$ and boxcrossing loss $(p t)$ in the performance in the dual condition in relation to the single condition is calculated as follows: $p m=p s-p d$ and $p t=(t s-t d) / t s$. The measure $m u$ is expressed in terms of a percentage of the performance in the single tasks, considering the contribu- 
tions of both tasks to be of equal weight: $m u=[1-(p m+p t) / 2] \times 100$.

Thirty-three healthy volunteers, university students, 17 women and 16 men, participated in this study. The participants ranged in age from 19 to 34 years, with a mean age of 25.67 years $(\mathrm{SD}=4.06)$.

In the verbal task, randomly determined sequences of digits were presented to the subject in a verbal-oral way and at the rate of 1 digit per second. The subject was required to repeat them in the proper order. The digit sequence length varied according to the subject's digit span. The subject's span was determined as the sequence length in which three sequences were repeated successfully. After the span determination, the subjects received digit sequences within a 2-min period. The dependent variable was the proportion of correct repeated sequences. In the visuospatial task, the subject was required to write an "X" with a felt tip pen on squares linked with arrows and aligned in an irregular way on a sheet of paper. Each sheet of paper contained 40 squares. Subjects were asked to fill the squares as fast as possible for a period of 2 min. If all boxes had been crossed before the time limit, a new sheet of boxes was presented. The dependent variable was the number of squares traversed with "Xs" within the limit of $2 \mathrm{~min}$. The dual task involved the combined execution of the two tasks within a 2-min period. The dependent variables were the same as in the single task, but in this case the differences between the performance of the subjects in the single and dual tasks were evaluated by the index $m u(19,22,31,32)$.

The maximal interval between sessions was 14 days and no more than 1 session per day was held. The mean interval was 3.16 $(\mathrm{SD}=1.82)$ days.

\section{Treatments A and B}

We designed two sets of digit sequences. In one set, we had two lists of sequences, one for the single task and the other for the dual task, which were used throughout the 20 sessions (treatment A). The other set was composed of 40 different lists, two per session (treatment B). In the first session we administered identical lists for both treatments A and B. Twenty-three subjects who performed the dual task in the usual order, first the single task and then the dual task, were assigned to two groups. Six women and five men received treatment $\mathrm{A}$, whereas six women and six men received treatment $\mathrm{B}$. Two men did not complete the study. Each of the remaining 21 subjects completed 20 sessions and responded to a questionnaire for qualitative evaluation during the last session, which asked about the task that received more attention in the dual condition, the easier and the harder task, and the strategies employed in the digit sequence recall.

The digit-span length determined in the first session was used in the first ten sessions. A preliminary analysis of the results obtained in these initial sessions showed that the subjects were performing the digit-span task without errors and effortlessly, and suggested that the digit-span length determined in the first session could have been underestimated. Span length was not expected to have an effect on $m u$ (19), so we decided to re-evaluate the digit-span length in the 11th session and change the span length of those subjects whose span lengths had increased in this second evaluation. These subjects received the new span length for the remaining ten sessions. In the data analysis, we took into account the span change by dividing the 20 sessions into two blocks, one comprising the first ten sessions and the other the remaining sessions. To assess the effect of practice, we evaluated again the span length in the last session.

In the first session, we informed the subjects about the characteristics of the tasks before determining the span length. After that, the subjects performed the single tasks. 
To ensure the correct understanding of the box-crossing task, we provided a practice trial with a sheet of paper containing a shorter chain with ten squares. Then, the subjects performed simultaneously the box-crossing task and the verbal task. In the remaining sessions, subjects performed only the single and dual tasks.

\section{Inverse treatment}

Subjects who performed the tasks in the inverse order (inverse treatment), five women and five men, received the same lists as the subjects who received treatment $\mathrm{A}$. These subjects completed five sessions and responded to the questionnaire for a qualitative evaluation in the last session. The digitspan length determined in the first session was used in the other four sessions. In the first session, subjects received information about the characteristics of the tasks and then the span length was determined. Subjects performed the practice trial of box- crossing, the dual task and then the digit and the box-crossing tasks separately.

\section{Results}

We first present the analysis of the data corresponding to the performance of the subjects who executed the tasks in the direct order (treatments A and B) throughout twenty sessions. We then present the analysis of the data corresponding to the performance of the subjects who performed the tasks in the inverse order throughout five sessions. The performance of the subjects who received the inverse treatment was compared with the performance of the subjects who received treatment A.

\section{Direct order}

The proportion of correct repeated digit sequences, the number of traversed squares and $m u$ were analyzed separately as a function of gender, treatment (A and B), task

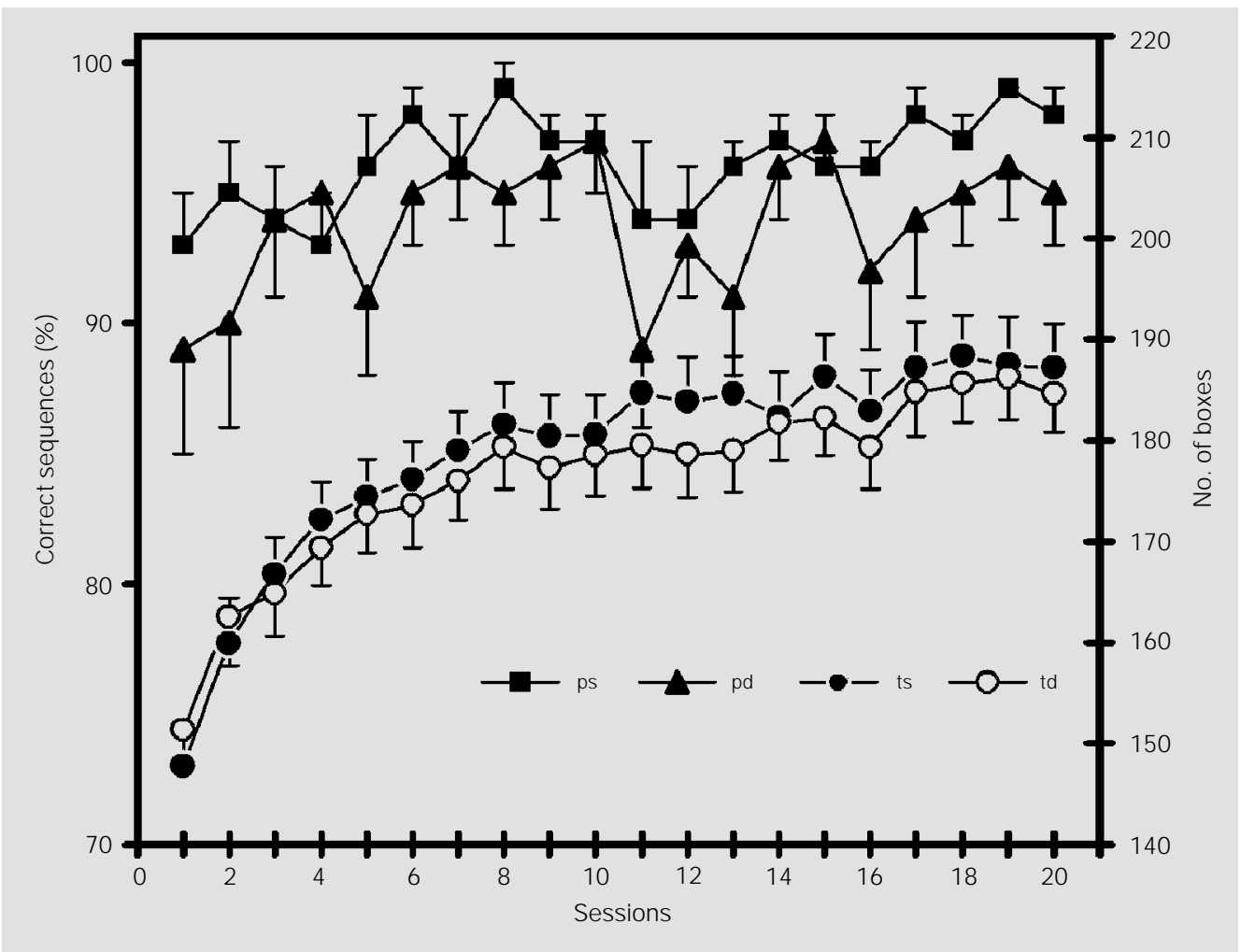

Figure 1 - Performance (mean \pm SEM) in the digit span and boxcrossing in the single and dual tasks when the single tasks were administered first $(\mathrm{N}=21)$; ps and pd are the proportion of correct repeated digit sequences under the single and dual conditions, respectively, expressed as percentage of the total number of presented sequences; ts and td are the number of traversed boxes in single and dual box-crossing tasks, respectively. 
condition (single and dual), block (the first ten sessions and the second ten sessions) and training (ten sessions), by analysis of variance. An alpha level of 0.05 was used for all statistical tests.

The means of the proportion of digit sequences correctly repeated in the single- (ps) and dual- $(p d)$ digit-span tasks, expressed as percentages for the 20 sessions, are presented in Figure 1. Data analysis showed that gender or treatment did not affect $p s$ or $p d$. The proportion of digit sequences retrieved was greater in the single-task condition, $\mathrm{F}(1,17)=6.61, \mathrm{P}$ $=0.02$, and increased with training, $F(9,153)=$ $5.51, \mathrm{P}<0.0001$. The improvement of performance due to training was greater in the first ten sessions. After the 10th session the performance in the digit task still improved, but at a lower rate. This trend was confirmed by the task condition $\mathrm{x}$ blocks $\mathrm{x}$ training interaction, $\mathrm{F}(9,153)=1.98, \mathrm{P}=0.046$.

Figure 1 also presents the mean number of traversed squares in the box-crossing task in the single- and dual-task conditions, $t s$ and $t d$, respectively. Gender, treatment and task condition, as main factors, did not affect the performance in single and dual box-crossing along the sessions. However, the number of traversed squares increased with training, $\mathrm{F}(9,153)=23.42, \mathrm{P}<0.0001$, and also increased in the second block, $F(1,17)=26.56$,
$\mathrm{P}<0.0001$. The interaction between these factors also suggests that there was a change in the gain in performance from the first to the second block of sessions, $F(9,153)=18.59$, $\mathrm{P}<0.0001$.

Whereas performance in the single tasks was affected by training, the measurements of loss in the simultaneous performance in the box-crossing ( $p t)$ and in the digit task (pm) were not affected by treatment or training. The interaction between training and block was significant for $p m, \mathrm{~F}(9,153)=$ $2.01, \mathrm{P}=0.042$, and for $p t, \mathrm{~F}(9,153)=1.95$, $\mathrm{P}=0.048$. In the first two sessions of the first block a better performance in the box-crossing task was observed in the dual condition, expressed as more negative $p t$ values, which had a compensatory effect on the $m u$ values with respect to the loss in the digit task. Figure 2 shows the relation between $\mathrm{pm}$ and $p t$ and its contributions to $m u$. The $m u$ values were affected only by the interaction between training and treatment, $F(9,153)=$ $2.30, P=0.019$. Duncan's multiple range test was performed and permitted to localize this effect to session 11, when some subjects changed their span length.

\section{Effect of the change in span}

The re-evaluation of span length in the
Figure 2 - Performance (mean \pm $\mathrm{SEM})$ in the dual task $(\mathrm{N}=21)$; $\mathrm{pm}$ and $\mathrm{pt}$ are the proportion of loss of performance in the dualdigit span and box-crossing tasks, respectively, with regard to the single tasks; $\mathrm{mu}$ is the composite measure of the simultaneous performance in the dual task. The negative values indicate gain in performance.

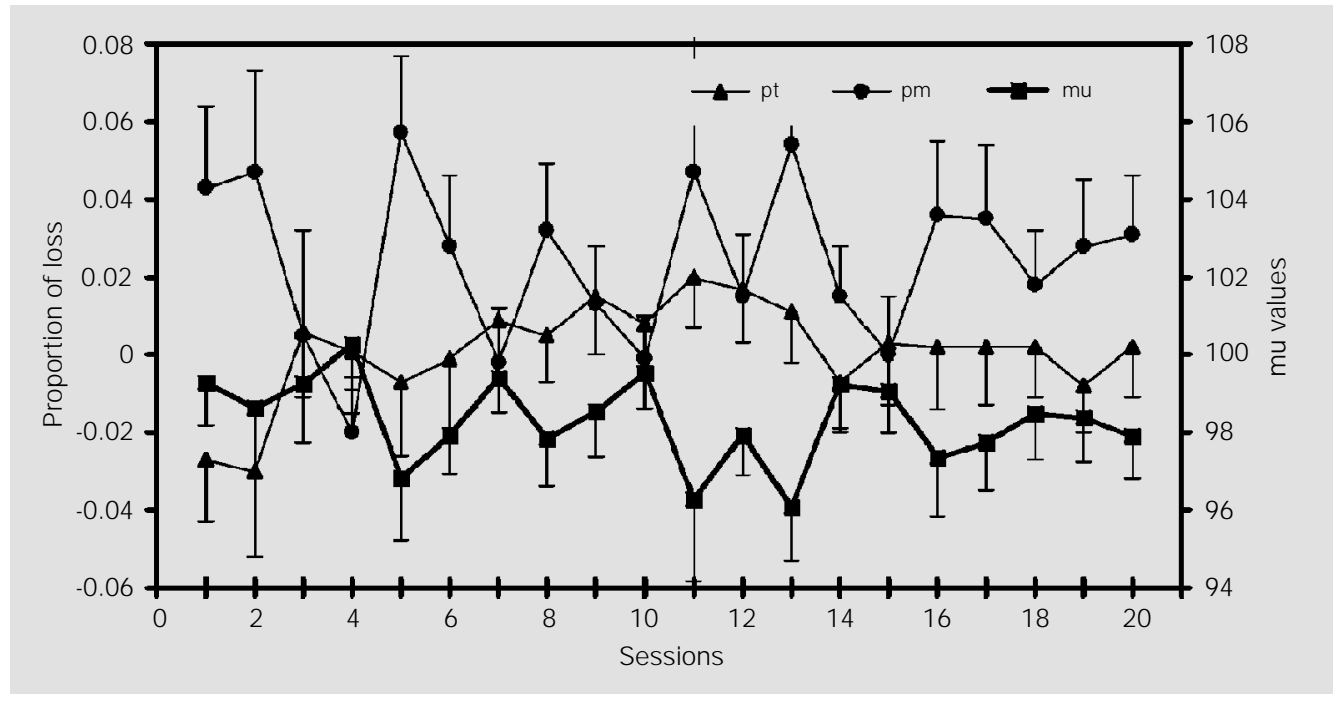


11 th session increased the mean span length of 8 subjects (three from treatment A and five from treatment $\mathrm{B}$ ), from 3.88 ( $\mathrm{SD}=$ $0.64)$ to $5.53(\mathrm{SD}=0.74)$. The mean span length of the other subjects, 4.77 (SD = 1.01 ), remained constant throughout the 20 sessions. Although an effect of span length on $m u$ values was not expected, the change in span introduced in the 11th session was accompanied by a decrease in $m u$ values. Thus, we analyzed the $m u$ values as a function of the span change, block and training to determine the potential contribution of the change in span length in session 11 , where five of ten subjects under treatment $B$ and three of 11 subjects under treatment $A$ showed an increased $\operatorname{span}^{1}$. A significant difference in the performance of the dual task was found in session 11 between the subjects who maintained the same span length along the 20 sessions and the subjects whose span increased in the 11th session. Data analysis showed an interaction between span change $\mathrm{x}$ block, $\mathrm{F}(1,19)=5.05, \mathrm{P}=0.037$, and span change $x$ block $x$ training, $F(9,171)$ $=2.49, \mathrm{P}=0.011$. The stability of the performance of subjects whose span length was constant throughout the 20 sessions can be observed in Figure 3. It can also be observed that the performance of eight subjects whose span length changed in the 11th session decreased from the first to the 11 th session and then increased to the level of the other group.

A separate analysis of the data obtained in the first session shows that the $m u$ values of the subjects whose span length changed in the 11th session were higher than the $m u$ values of the subjects who worked with the same span length throughout the 20 sessions. Although this difference between $m u$ values was not significant, $F(1,19)=4.02$, $P$ $=0.059$, it suggested a negative correlation between $m u$ values and span length. Indeed, the Spearman correlation coefficient between $m u$ values and span length for the first session was $-0.643, \mathrm{P}<0.001$. Span length was significantly correlated only with the $\mathrm{pm}$ component of $m u, r=0.650, \mathrm{P}<0.001$.

The reliability of the $m u$ index, estimated by the Pearson correlation coefficient between the $m u$ obtained by 22 subjects in the first and the second session, was $0.577, \mathrm{P}=$ 0.006 .

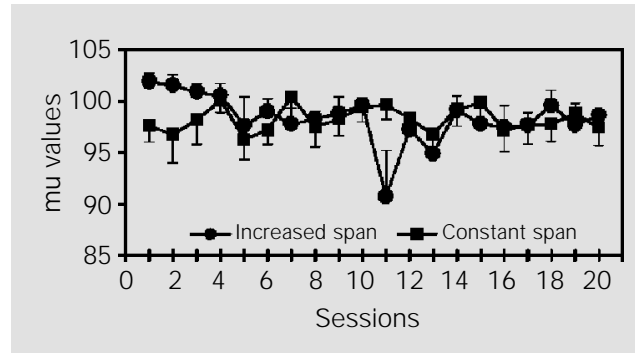

Figure 3 - Performance (mean \pm $\mathrm{SEM}$ ) in the dual task, expressed by the composite measure mu, for the eight subjects whose span length increased in session 11 and for the 13 subjects whose spans were constant throughout the 20 sessions.

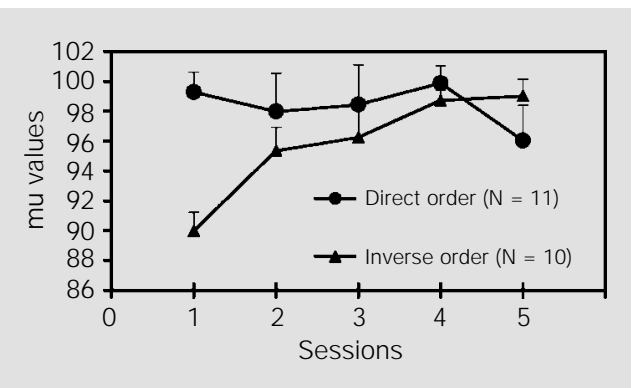

Figure 4 - Performance (mean \pm SEM) in the dual task as a function of the order of task administration, expressed as mu values.
Table 1 - Performance in box-crossing tasks (No. of boxes).

Data are reported as means $\pm \mathrm{SD}$. $* \mathrm{P}<0.0002$ (Duncan's multiple range test following three-way ANOVA).

\begin{tabular}{lccccc}
\hline Session & \multicolumn{2}{c}{ Direct order $(\mathrm{N}=11)$} & & \multicolumn{2}{c}{ Inverse order $(\mathrm{N}=10)$} \\
\cline { 2 - 3 } \cline { 5 - 6 } & Single task & Dual task & & Single task & Dual task \\
\hline 1 & $148.7 \pm 20.1$ & $154.2 \pm 19.2$ & & $159.9 \pm 18.0$ & $132.6 \pm 22.5^{*}$ \\
2 & $164.0 \pm 19.0$ & $166.2 \pm 27.1$ & & $166.8 \pm 18.4$ & $156.9 \pm 23.5$ \\
3 & $167.6 \pm 20.5$ & $163.5 \pm 24.3$ & & $173.0 \pm 20.1$ & $164.3 \pm 22.4$ \\
4 & $173.7 \pm 18.4$ & $171.1 \pm 19.6$ & & $178.3 \pm 19.4$ & $173.2 \pm 19.6$ \\
5 & $175.1 \pm 19.9$ & $171.2 \pm 19.4$ & & $178.8 \pm 19.7$ & $175.2 \pm 18.1$
\end{tabular}

${ }^{1}$ The assignment of the subjects to the treatments did not take into account their span length. Although the difference in the mean span length between treatments $A$ and $B$, as determined in the first session, was not significant $\left(t_{(19)}=1.028, P=0.159\right)$, the two subjects with the smallest span length (three digits) received treatment $B$ and the subject with the largest span length (seven digits) received treatment $A$. So, there was an unequal distribution of span length and span changes for both treatments. 
The mean span lengths obtained for the 21 subjects in sessions 1,11 and 20 were $4.43(\mathrm{SD}=0.98), 4.86(\mathrm{SD}=1.01)$ and 5.48 $(\mathrm{SD}=1.17)$, respectively, but no correlation was found between the span length obtained in these sessions. Gender or treatment did not affect the changes in span length, but the within-subject span length variation was significant, $\mathrm{F}(2,34)=5.57, \mathrm{P}=0.008$.

\section{Order of administration}

To assess the effect of order of administration, the values of $m u$ and its components, $p m$ and $p t$, were analyzed separately as a function of gender, order of administration (direct and inverse) and training (five sessions) by analysis of variance. An alpha level of 0.05 was used for all statistical tests. The $p t$ values were affected by order of task administration, $\mathrm{F}(1,17)=11.64, \mathrm{P}=0.003$, and by the training $\mathrm{x}$ order of administration interaction, $\mathrm{F}(4,68)=10.21, \mathrm{P}<0.0001$. Duncan's multiple range test permitted us to localize a large loss in the box-crossing performance only in the first session under the inverse order. The $\mathrm{pm}$ values were not significantly affected by gender, order of administration or training factors.

Figure 4 presents the mean $m u$ values obtained for both groups for the five sessions. Analysis of the $m u$ values showed a main effect of training, $\mathrm{F}(4,68)=3.42, \mathrm{P}=$ 0.013 , and an interaction between training $\mathrm{X}$ order of administration, $\mathrm{F}(4,68)=4.43, \mathrm{P}=$

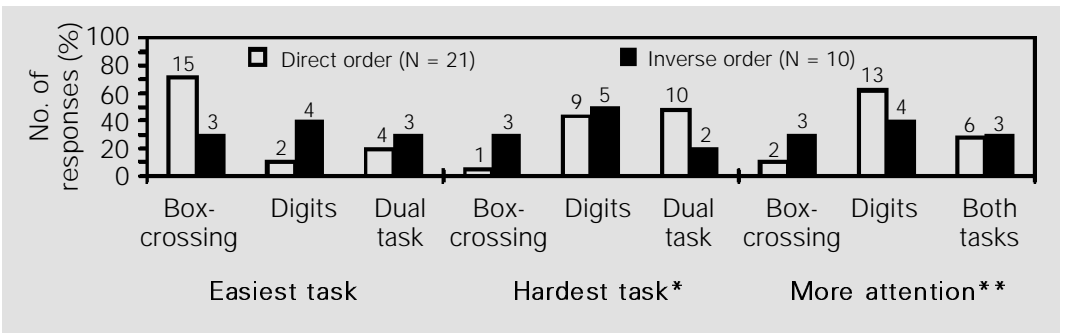

Figure 5 - Percentage of responses to the questionnaire distributed by the order of task administration. The digits over the bars indicate the numbers of subjects for that response. *One subject could not say which task was the hardest. **Refers to the task that received more attention under the dual condition.
0.003. The post hoc Duncan's multiple range test showed this effect only for the first session under the inverse order condition. Table 1 shows the mean number of traversed squares in the single and dual task under the direct and inverse order of administration for the five sessions. Taken together, these findings support the idea that the poorer performance in the dual task was due to a smaller number of traversed squares in the dual box-crossing when the dual task was administered before the single tasks, affecting $m u$.

The span length for the subjects who executed the task under the inverse order ranged from 3 to 5 , with a mean of $4.3(\mathrm{SD}=$ 0.82 ). Again a significant negative correlation was found between the span length and the $m u$ values obtained for the first session, $\mathrm{r}=-0.869, \mathrm{P}=0.001$.

\section{Responses to the questionnaire}

Figure 5 exhibits the responses of 31 subjects who participated in both studies to the final questionnaire, grouped by the order of task administration. Qualitative analysis showed some differences between groups. The majority of subjects who executed the tasks in the direct order reported that boxcrossing was the easiest task and was the least indicated as the task that received more attention in the dual condition because "it can be executed automatically". On the other hand, the group that executed the tasks in the inverse order showed more equity in response distribution. In general, the dual task has been considered to help against the dispersion of attention. Subjects reported that, during the single-digit span task, it was difficult to focus attention on the digit-sequence chain.

The strategies reported by the subjects were grouped as a function of the required effort. Thus, "just listen and repeat", "to repeat by the sound" and "not using strategies at all" were differentiated from "to imag- 
ine the numbers", "to group the numbers", "to hold only the first numbers letting the last ones come by themselves" or "to make associations". We assumed that this last group of reported strategies would require a higher level of attention effort. The subjects who increased their span length reported changes in their strategies. Only those subjects who obtained a span length up to 4 digits reported the "effortless" strategies, whereas the more effort-requiring strategies were reported when the span length was of 4 digits or more.

\section{Discussion}

Our objective was to study the $m u$ index (19), which is supposed to indicate the coordination capacity in the dual-task executive test proposed by Della Sala et al. (22). We studied the influence of training on the performance in the dual task throughout 20 sessions, the effect of the repetition of the same digit sequence lists or always-different lists in the memory task, and the contribution of the within-session practice to the $m u$ index by changing the order of execution of the tasks.

The performance in the isolated tasks increased with training, but the coordination capacity remained stable. We found a significant correlation between span length and $m u$. Also, we found that the capacity to coordinate the simultaneous execution of both tasks was better under the direct order due to the within-session practice in the single box-crossing. The reported strategies suggest that the perceived task difficulties seem to be related to the order of the tasks. Some of the results obtained in the present study agree with those obtained by Baddeley et al. (19). Gender did not affect the mu values. The level of reliability of the $m u$ values for the first and second session was very similar and also considered low for the clinical use of the test, although the intervals between sessions differed from those of the normative study (19).
Our results show that the improved performance in the tasks under the dual condition closely resembled the improvement in the single-task performance. So, although training affected performance in the single and dual conditions, especially for the tracking component, the $m u$ values remained stable throughout the sessions when the single tasks were performed first. We expected the regular repetition of a set of items from session to session to result in an improvement in the recall of those items (Hebb, 1961, cited in Ref. 28). However, no significant difference was observed in the performance of subjects who received same digit sequence lists compared to subjects who received always different lists. But we observed that some subjects could remember the first sequences spontaneously after few sessions. So, it is possible that the method used was not sensitive enough to detect the effect of the sequence repetition. It is important to note that the practice effects with the same digit sequence lists throughout sessions are relevant to the clinical use of the test in patient follow-up.

The statistically significant interaction between training and the similarity of digit sequence lists that affected the $m u$ values specifically in session 11 may be attributed to an increase in span length. Span length variability was recorded not only between subjects, but also within subjects. For the first session, $m u$ and the span length showed a negative correlation. Subjects showed variability of their span lengths, as evaluated in sessions 1, 11 and 20. It seems that an increase in span length affects the performance in the dual task. The span measure was taken as the maximum length at which the subject performed all three lists successfully. This method, proposed in a previous study of the dual task (22), allows obtaining a more conservative measure with respect to the standard tests of intellectual ability (33). Since the variation observed in span length directly affects $m u$, research about methods 
for determining the span length for this task acquires new relevance. We propose that the differential effects between more exacting and more conservative span measures on performance in dual tasks should be taken into account (34-36).

The strategies reported by the subjects seem to be affected by span length. Logie et al. (37), taking into account the phonological similarity and word length effects, suggested the involvement of PL only when verbal rehearsal or chunking strategies were reported, whereas other strategies, e.g., visual mnemonic ones, do not necessarily rely on the use of verbal STM. The increase in the number of items seems to involve strategies with other resources in addition to those proposed for the PL. These supplementary strategies to retain more items in a nonacoustic way might involve resources from the visual or semantic component of STM (9), or from the focus of attention through the CE (11,38,39). Cowan et al. (11) also proposed that the $\mathrm{CE}$ contributes to the differences in memory span when rehearsal strategies that would require repeated planning and management are used.

It is possible that some strategies constructed when the single tasks are performed first may have a heavier load in the dual-task condition, and in this case these strategies are usually changed due to a possible expensive cost-benefit relation (40), as was reflected in the reports by some subjects in the present study. Since the differential use of strategies may affect the performance (40), the test-retest and the between-subjects reliability (37), we agree with Logie et al. (37) with respect to the relevance of the reported strategies for the interpretation of the data. In the present study, we divided the reported strategies into two groups as a function of the required effort in the attempt to retain and recall the digits from each sequence. Subjects who employed strategies that required a higher-level of attention effort (e.g., involving chunking, holding just the first digits, forming associations between digits or generating visual images of them), obtained a span length of 4 digits or more and their $m u$ values tended to be smallest in the first session. On the other hand, subjects with strategies that required minor or no effort at all (e.g., just listening and repeating by the sound or not using strategies at all) obtained the smallest span and the highest $m u$ values, close to or over $100 \%$. Since strategies were required only in the final session and subjects reported changes in the employed strategies, the relations between the reported strategies and $m u$ should be considered with caution. Nevertheless, in a further study (35) we manipulated the span length and the results obtained also suggest the involvement of the strategies in the digit span on $m u$.

Several issues about the strategies in memory task might be pointed out; e.g., do the effort-requiring strategies rely on executive capacities? When those strategies are used which of the tasks is more affected under the dual-task condition? Vandierendock et al. (36) found that the memory performance in a visual forward-memory-span task is not affected by the concurrent execution of a random interval tapping task, which supposedly interferes with executive functions. They concluded that $\mathrm{CE}$ is not involved in the visual forward-digit-span task. Although it was not systematically related to recall performance, the tapping task performance was poorer under the dual- than under the single-task condition. This result suggests, in agreement with other studies $(16,18)$, that the performance in a motor task will be more affected as the amount of information stored in a concurrent memory task increases. When the performance in the motor task is impaired in the dual condition, we may speculate that this occurs because the $\mathrm{CE}$ is involved in the effort-requiring strategies.

The interference of a verbal task, e.g., digit load or articulatory suppression, with a concurrent non-verbal task suggests a de- 
mand on more general processing resources, in addition to demands on specifically phonological resources. Teasdale et al. (30) suggest that the attentional control of action is associated with conscious awareness. Indeed, in our study some subjects reported that on several occasions they lost the conscious awareness of the tasks they were performing. This situation was not generally realized by the experimenters because the subjects performed normally. It should be pointed out that the flowing of thoughts not related to the tasks was reported mainly for the single box-crossing task. For the verbal task, only subjects whose verbal task strategies were effortless reported the flowing of thoughts. So, the high conscious awareness of subjects with effort-requiring strategies in the singleverbal task may be related to $\mathrm{CE}$ requirements.

Although $m u$ seems not to be affected by training along sessions, the execution of a dual task first affected significantly the performance in the first session, under the novelty of the condition. Thus, practice could have been affecting the performance when the dual task was administered after the single task; some of the better performance in the dual task can be explained in this way (19). Dual-task performance changes as a function of practice, but practice in single tasks seems not to be sufficient for the best performance in the dual task. In this case, additional dual-task training is needed. The singletask practice facilitates the development of strategies that could be fast and accurate, but with a variable degree of resource consumption. On the other hand, multitask training facilitates the development of optimal combined strategies for performing all of the tasks, e.g., by delaying tasks, adopting lowworkload strategies and chunking transmission $(27,29,40)$. It is also possible that the single-task training in tracking tasks is transferred to the dual condition through the development of higher-order control mechanisms, subsequently allowing automatic pro- cesses to function without controlled processing, with a reduction in processing time and effort (29). Probably the effect of prior practice is mediated by reductions in demands for central resources common to the two tasks, rather than reductions of specialized verbal or visuospatial processing resources in which the two tasks differ (30).

Demands on the control and coordinating functions of CE may be greater when the tasks are novel than when the dual task follows the single tasks. In the present study, subjects that performed the single tasks first had a better performance in the dual task than subjects that executed the tasks under the inverse order. These subjects had a lower performance in dual box-crossing task. This result raises the question of whether $m u$, a measure of task coordination capacity, could be confounded with a within-session practice effect. The $m u$ index reflects the mean loss in performance in both tasks under the dual condition, but does not permit differentiating between the contributions of the visuospatial and verbal components. Our results suggest that the effect of the withinsession practice in the first session on $m u$ could be attributed more to the box-crossing task than to the digit task. In this way the same $m u$ value can be obtained by performing each task at different levels, e.g., a better performance in the dual box-crossing could mask a worse performance in the dual-digit task.

The use of effort-requiring strategies under the dual condition may lead to a diminished performance in the tracking task in order to maintain the performance in the dual verbal task at the level of the single condition. Strategies of attention distribution seem to be related to the order of the tasks since subjects who performed the two concurrent tasks first reported a more balanced distribution of the attention between the two tasks than subjects who performed the single tasks first did. The differences 
between strategies of attention distribution may be dependent on the condition that was performed first, i.e., single or dual, since the encoding demands may differ between learning conditions (27).

The number of filled squares in the boxcrossing task within the two-minute period includes both accuracy and velocity components. The performance in the digit-span task is measured only in terms of accuracy, i.e., number of correct repeated sequences. We consider that the velocity component in the digit-span task should also be taken as another factor in evaluating the performance in the dual task since the contributions of both tasks are supposed to be of equal weight on $m u$. On the basis of this assumption, we compared the total number of sequences recalled in the dual condition with the total number of sequences recalled in the single condition without considering errors. Then, we determined a velocity measure in the digit-span task using the following formula: $(N s-N d) / N s$. The velocity in digit-span performance of the group of subjects who performed the task under the inverse order was compared with the velocity in digit-span performance of the group of subjects who received treatment A. Although the order of the tasks did not affect the accuracy in sequence recall, the total number of presented sequences was lower under the inverse order and was higher under the direct order only in the first session, $\mathrm{F}(4,76)=5.49, \mathrm{P}<0.001$. Cowan et al. (11) proposed a model that includes two separate verbal processing rates, i.e., the rapid articulation and the retrieval of words from STM, which may contribute to memory span. The speeded articulation may serve as an index of the efficiency of PL, whereas preparatory intervals in the spantask responses and pauses between items to be recalled may reflect the efficiency of CE functioning. The decrease in velocity in the dual digit-span task is more evident in some frontal patients' performance. So, although some timing measures are not easily interpretable (11), we believe that further research about velocity components in the recall of digit sequences may be promising for the elucidation of the mechanisms involved in performance of single and dual tasks.

It is undoubtedly important to have a reliable clinical test to assess the dysexecutive syndrome and the present dual task, proposed by Della Sala et al. (22), is a very promising tool whose reliability should be improved. The negative correlation between span length and $m u$ that we found creates noise about the clinical interpretation of the performance in this dual task. As an example of this we may cite the case of a patient evaluated with the present dual task, a 59year-old woman who underwent surgery to extirpate a meningeal frontal left tumor. Before surgery, her span length was determined as two digits and her ability for dual-task coordination, as measured by the $m u$ index, was 96.36. In a follow-up session six months later, she attained a span length of three digits and a $m u$ index of 79.2. It is difficult to interpret this reduction in the capacity for coordination, expressed by $m u$, together with the increase in span length if we do not have a clinical cut-off point or a clear knowledge of the contribution of span length to the coordination capacity. Further research is necessary to improve the reliability of the measures proposed for this dual task, which is a quite promising tool for clinical practice and provides interesting insights into the executive processes.

\section{Acknowledgments}

We thank Prof. A. Baddeley for providing a copy of the current version of the dualtask test and the names of three anonymous reviewers for their helpful comments on an earlier draft of this paper. 


\section{References}

1. Baddeley AD \& Hitch GJ (1974). Working memory. In: Bower GH (Editor), The Psychology of Learning and Motivation. Academic Press, New York, 47-90.

2. Baddeley AD (1996). Exploring the central executive. Quarterly J ournal of Experimental Psychology, 49A: 5-28.

3. Baddeley AD (1994). Working memory: the interface between memory and cognition. In: SchacterDL \& Tulving E (Editors), Memory Systems. MIT Press, Cambridge, MA.

4. Baddeley AD (1986). Su Memoria: Cómo Conocerla y Dominarla. Debate, Buenos Aires, 172-189.

5. Baddeley AD (1986). Working Memory. Oxford University Press, Oxford.

6. Vecchi T, Monticellai ML \& Comoldi C (1995). Visuospatial working memory: structures and variables affecting a capacity measure. Neuropsychologia, 33: 1549-1564.

7. Richardson J TE (1996). Evolving issues in working memory. In: Richardson JTE, Engle R, Hasher L, Logie R, Stoltzfus E \& Zacks R (Editors), Working Memory and Cognition. Oxford University Press, New York.

8. Smith DJ , Wilson M \& Reisberg D (1995). The role of subvocalization in auditory imagery. Neuropsychologia, 33: 1433-1454.

9. Zhang G \& Simon HA (1985). STM capacity for Chinese words and idioms: chunking and acoustical loop hypotheses. Memory and Cognition, 13: 193-201.

10. McCarthy RA \& Warrington EK (1990). Cognitive Neuropsychology. A Clinical Introduction. Chapter 13. Academic Press, California.

11. Cowan N, Wood NL, Wood PK, Keller TA, Nugent LD \& Keller CV (1998). Two separate verbal processing rates contributing to short-term memory span. J ournal of Experimental Psychology: General, 127: 141-160.

12. Anderson B (1975). Cognitive Psychology. The Study of Knowing, Learning and Thinking. Academic Press, New York.

13. Dubois $B$, Levy $R$, Verin $M$, Teixeira $C$, Agid $Y$ \& Pillion B (1995). Experimental approach to prefrontal functions in humans. Annals of the New York Academy of Sciences, 769: 41-60.

14. Lehto J (1996). Are executive function tests dependent on working memory capacity? Quarterly J ournal of Experimental Psychology, 49A: 29-50.

15. Logie RH, Gilhooly KJ \& Wynn V (1994). Counting on working memory in arithmetic problem solving. Memory and Cognition, 22: 395-410.
16. Shulman HG \& Greenberg SN (1971). Perceptual deficit due to division of attention between memory and perception. J ournal of Experimental Psychology, 88: 171176.

17. Stephaneck P (1972). Algumas contribuições experimentais ao problema da avaliação da carga do trabalho não físico. Doctoral thesis, University of São Paulo, Ribeirão Preto, SP, Brazil.

18. J ohnston WA, Greenberg SN, Fisher RP \& Martin DW (1970). Divided attention: a vehicle for monitoring memory processes. J ournal of Experimental Psychology, 83: 164-171.

19. Baddeley $A D$, Della Sala $S$, Gray $C$, Papagno C \& Spinnler H (1997). Testing central executive functioning with a pencil-and-paper test. In: Rabbitt P (Editor), Methodology of Frontal and Executive Functions. Chapter 3. Psychology Press, Hove, UK.

20. Baddeley AD, Bressi S, Della Sala S, Logie $\mathrm{R} \&$ Spinnler H (1991). The decline of working memory in Alzheimer's disease. A longitudinal study. Brain, 114: 2521-2542.

21. Baddeley $A D$, Logie R, Bressi S, Della Sala S \& Spinnler H (1986). Dementia and working memory. Quarterly J ournal of Experimental Psychology, 38A: 603-618.

22. Della Sala S, Baddeley AD, Papagno C \& Spinnler H (1995). Dual task paradigm: a means to examine the central executive. Annals of the New York Academy of Sciences, 769: 161-171.

23. Greene J DW, Hodges J R \& Baddeley AD (1995). Autobiographical memory and executive function in early dementia of Alzheimer type. Neuropsychologia, 33: 16471670.

24. Heuer H (1996). Dual-task performance. In: Neumann $O \&$ Sanders $F$ (Editors), Handbook of Perception and Action. Vol. 3. Attention. Academic Press, London.

25. Underwood G \& EverattJ (1996). Automatic and controlled information processing: the role of attention in the processing of novelty. In: Neumann $O$ \& Sanders F (Editors), Handbook of Perception and Action. Vol. 3. Attention. Academic Press, London.

26. Logan G (1988). Automaticity, resources, and memory: theoretical controversies and practical implications. Human Factors, 30: 583-598.

27. Detweiler M \& Lundy D (1995). Effects of single- and dual-task practice on acquiring dual-task skill. Human Factors, 37: 193-211.

28. Schwartz M \& Bryden MP (1971). Coding factors in the learning of repeated digit sequences. J ournal of Experimental Psychology, 87: 331-334.

29. Schneider W \& Detweiler M (1988). The role of practice in dual-task performance: toward workload modeling in a connectionist/control architecture. Human Factors, 30: 539-566.

30. Teasdale J D, Dritschel BH, Taylor MJ, Proctor L, Lloyd CA, Smith IN \& Baddeley AD (1995). Stimulus-independent thought depends on central executive resources. Memory and Cognition, 23: 551-559.

31. Baddeley AD, Della Sala S, Papagno C \& Spinnler H (1997). Dual-task performance in dysexecutive and nondysexecutive patients with a frontal lesion. Neuropsychology, 11: 187-194.

32. Wilson B (1995). Memory and executive functioning: theory, assessment and treatment. Symposium conducted at the Meeting of the "Simposio sobre Memoria y Amnesias" organized by the Sociedad Neuropsicológica Argentina, Buenos Aires.

33. Wechsler D (1995). Test de Inteligencia para Adultos (WAIS). Translated by Álvarez HF. Paidós, Buenos Aires (Original work published in 1955).

34. Baddeley AD, Emslie H, Kolodny J \& Duncan J (1998). Random generation and the executive control of working memory. Quarterly J ournal of Experimental Psychology, 51A: 819-852.

35. Sylwan RP, Rosin FM \& Galera C (1999). Effect of practice and span length on the dual-task coordination executive test. Brazilian J ournal of Medical and Biological Research, 32: 1263-1268.

36. Vandierendock A, De Vooght $G \&$ Van der Goten K (1998). Interfering with the central executive by means of a random interval repetition task. Quarterly J ournal of Experimental Psychology, 51A: 197-218.

37. Logie RH, Della Sala S, Laiacona $M$, Chalmers P \& Wynn V (1996). Group aggregates and individual reliability: the case of verbal short-term memory. Memory and Cognition, 24: 305-321.

38. Cowan N (1994). Mechanisms of verbal short-term memory. Current Directions in Psychological Science, 3: 185-189.

39. Cowan N (1995). Attention and Memory: An Integrated Framework. Oxford Psychology Series, No. 26. Oxford University Press, New York.

40. Logan GD, Zbrodoff NJ \& Fostey ARW (1983). Costs and benefits of strategy construction in a speeded discrimination task. Memory and Cognition, 11: 485-493. 